\title{
Concurrent Small-Cell Transformation and Emergence of Trans-C797S and T790M Mutations Under Sequential Treatment With EGFR Inhibitors in Lung Adenocarcinoma
}

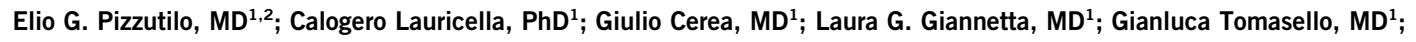
Stefano Stabile, PhD ${ }^{1}$; Valentina Motta, PhD ${ }^{1}$; Spyridon Alexiadis, MD ${ }^{1}$; Francesco Scaglione, MD, PhD ${ }^{1,2}$; Angelo Vanzulli, MD ${ }^{1,2}$; Massimo Torre, MD ${ }^{1}$; Emanuela Bonoldi, MD ${ }^{1}$; Silvio M. Veronese, PhD ${ }^{1}$; Salvatore Siena, MD ${ }^{1,2}$; and Andrea Sartore-Bianchi, MD ${ }^{1,2}$

\section{INTRODUCTION}

Epidermal growth factor receptor (EGFR) gene mutations are strong oncogenic drivers in a subset of non-small-cell lung cancer (NSCLC). Their inhibition with specific tyrosine kinase inhibitors (TKIs) represents a successful example of precision medicine. ${ }^{1}$ Nevertheless, disease progression almost invariably occurs after 9-14 months of treatment with either gefitinib, erlotinib, or afatinib (first- and second-generation TKIs) ${ }^{2-4}$ and after 19 months with osimertinib (a thirdgeneration TKI ${ }^{5}$ because of the development of acquired therapeutic resistance.

Several mechanisms of TKI resistance have been reported, with different mechanisms for first-, second-, ${ }^{6}$ and third-generation TKIs. ${ }^{7}$ The acquisition of T790M mutation in exon 20 of EGFR represents the most frequent mechanism (more than $50 \%$ of patients) of disease progression with gefitinib, erlotinib, or afatinib, which can be overcome by osimertinib and other thirdgeneration inhibitors. However, a frequent cause of third-generation TKI resistance is the development of mutations at the EGFR C797 codon, because they prevent binding to the EGFR active site. ${ }^{8,9}$ In these patients, preclinical studies have shown that the C797S mutation can be found in the same (in cis) or different (in trans) T790M-mutated alleles, or in other patients the T790M mutation can be lost. ${ }^{10}$

In clinical practice, resistant mechanisms to different TKIs can be detected on disease progression through tissue rebiopsy. The isolation and analysis of circulating tumor DNA (ctDNA) through liquid biopsy is also recommended and widely used for identification of acquired resistance mutations of EGFR. ${ }^{11}$

However, not only target-dependent resistance mechanisms can occur. Other mechanisms involve bypass pathways, such as MET amplification, human epidermal growth factor receptor 2 amplification, PIK3CA mutation, and BRAF mutation. ${ }^{12}$ Furthermore, epithelial to mesenchymal transition or histologic transformation to both squamous cell cancer ${ }^{13}$ and small-cell lung cancer (SCLC; in 3\%-15\% of patients) have been described after treatment with first-, second-, ${ }^{14,15}$ and third-generation TKIs. ${ }^{7,16}$

\section{CLINICAL COURSE}

A 75-year-old, nonsmoking white woman was diagnosed with stage IV lung adenocarcinoma in February 2017, with bilateral pulmonary lesions (Data Supplement), pleural effusion, and metastases to the thoracic lymph nodes, liver, adrenal gland, and bone. Based on an activating EGFR mutation (exon 19 deletion [del19]) detected from bronchoalveolar lavage cell block (Fig 1A), treatment consisted of gefitinib, which led to objective partial response of all lesions (Data Supplement). After 9 months, the patient experienced disease progression in the liver (Data Supplement), and a liquid biopsy revealed the acquisition of EGFR T790M mutation (Fig 1B; Table 1). She was then treated with osimertinib, obtaining stable disease with shrinkage of hepatic lesions (Data Supplement). Almost 12 months later, dyspnea worsened, with evidence of increased right pleural effusion and disease progression in the lung, lymph nodes, and liver (Data Supplement). A new liquid biopsy identified persistence of T790M plus the acquisition of C797S in trans conformation (Table 1; Fig 1-C). Two right thoracenteses and a talc pleurodesis were subsequently performed for treatment of recurrent pleural effusion; pleural biopsy revealed the presence of both adenocarcinoma and SCLC (Fig 1C), the latter with a high proliferation index (MIB-1, 100\%). We also found neuroendocrine cells in pleural effusion, which continued to harbor the original EGFR exon del19, without T790M or C797S mutations (Table 1). Molecular analysis of both ctDNA in pleural fluid and pleural biopsy showed the coexistence of del19, T790M, and in trans-C797S, consistently with the simultaneous presence of SCLC and NSCLC in the tissue sample (Table 1).

The patient started systemic therapy with carboplatin monotherapy in January 2019, but a pulmonary 


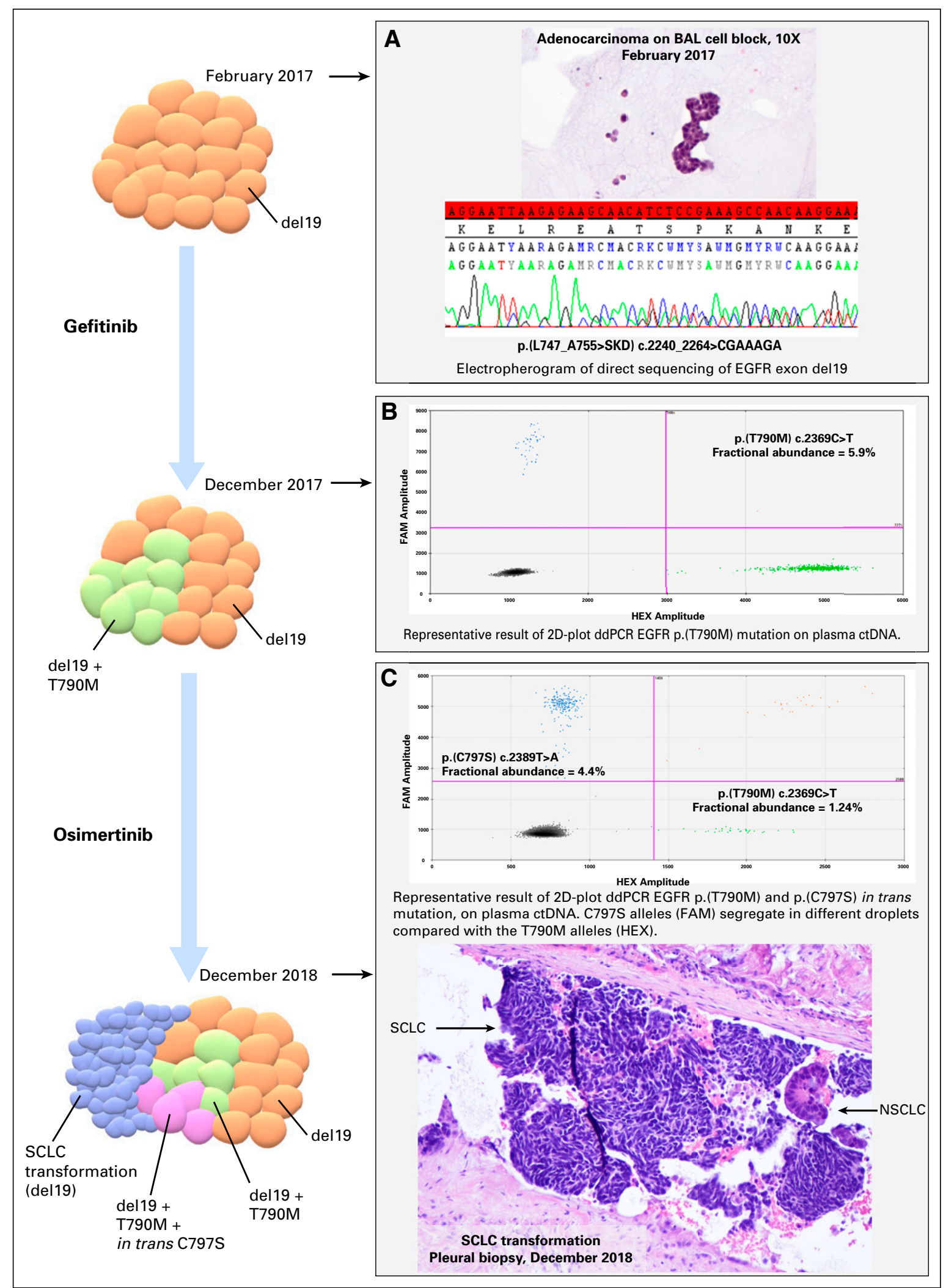

FIG 1. Synopsis of therapeutic history and histologic and molecular analysis of the present patient showing tumor evolution toward small-cell lung cancer (SCLC) transformation and accumulation of epidermal growth factor receptor (EGFR) genetic abnormalities. (A) Diagnosis of adenocarcinoma and detection of EGFR exon 19 deletion (del19). (B) Acquisition of T790M mutation. (C) Acquisition of C797S mutation on circulating tumor DNA (ctDNA) and evidence of SCLC transformation on pleural biopsy. BAL, bronchoalveolar lavage; ddPCR, droplet digital polymerase chain react; NSCLC, non-small-cell lung cancer. 
TABLE 1. EGFR Mutational Status by ddPCR Analysis in Different Samples

\begin{tabular}{|c|c|c|c|c|c|}
\hline Sample & $\begin{array}{c}\text { EGFR Exon del19 } \\
\text { p.(L747_A755>SKD) } \\
\text { c.2240_2264>CGAAAGA }\end{array}$ & $\begin{array}{c}\text { EGFR Exon } 20 \\
\text { p. }(\mathrm{T} 790 \mathrm{M}) \mathrm{c} .2369 \mathrm{C}>\mathrm{T}\end{array}$ & $\begin{array}{c}\text { EGFR Exon } 20 \\
\text { p.(C797S) in trans } \\
\text { c.2389T }>\mathrm{A}\end{array}$ & $\begin{array}{c}\text { T790M/del19 } \\
\text { Ratio }\end{array}$ & $\begin{array}{c}\text { C797S/del19 } \\
\text { Ratio }\end{array}$ \\
\hline \multicolumn{6}{|c|}{ Before Osimertinib } \\
\hline Plasma (ctDNA) & Positive & Positive & Negative & 0.24 & l \\
\hline December 2017 & FA: $24.8 \%$ & FA: $5.9 \%$ & & & \\
\hline \multicolumn{6}{|c|}{ After Osimertinib } \\
\hline $\begin{array}{l}\text { Cell block: neuroendocrine } \\
\text { cells in pleural fluid } \\
\text { December } 2018\end{array}$ & $\begin{array}{l}\text { Positive } \\
\text { FA: } 54.5 \%\end{array}$ & Negative & Negative & I & l \\
\hline Plasma (ctDNA) & Positive & Positive & Positive & 0.02 & 0.07 \\
\hline December 2018 & FA: $59.7 \%$ & FA: $1.24 \%$ & FA: $4.4 \%$ & & \\
\hline Pleural fluid (ctDNA) & Positive & Positive & Positive & 0.003 & 0.005 \\
\hline December 2018 & FA: $58.2 \%$ & FA: $0.2 \%$ & FA: $0.3 \%$ & & \\
\hline Pleural tissue & Positive & Positive & Positive & 0.03 & 0.004 \\
\hline December 2018 & FA: $42 \%$ & FA: $1.31 \%$ & FA: $0.2 \%$ & & \\
\hline
\end{tabular}

Abbreviations: ctDNA, circulating tumor DNA; ddPCR, droplet digital polymerase chain reaction; del, deletion; EGFR, epidermal growth factor receptor; FA, fractional abundance.

embolism occurred 6 weeks later, and she died despite thrombolytic therapy.

\section{DISCUSSION}

In EGFR-mutated NSCLC, the development of mutations at the EGFR C797 codon, which usually occurs in cis with T790M and in $<5 \%$ of patients in trans, ${ }^{9,16,17}$ is a frequent mechanism of resistance to third-generation TKIs because it prevents the drug from binding to the EGFR active site. Under therapeutic pressure exerted by osimertinib, it occurs in up to $24 \%$ of patients. ${ }^{17}$

Because the $\mathrm{C} 797 \mathrm{~S}$ mutation does not induce resistance to first-generation TKIs, a rechallenge with first-generation TKIs in combination with a third-generation TKI could be effective if the two mutations are in trans conformation ${ }^{10}$ so they do not coexist in the same receptor (mutations in different alleles or different clones). ${ }^{18}$ However, two case reports have demonstrated limited clinical benefit (progression-free survival [PFS] $<3$ months), ${ }^{19,20}$ and another report showed a PFS of 8 months with TKI combination treatment in addition to bevacizumab. ${ }^{21}$ Ongoing clinical trials are also exploring this therapeutic option (ClinicalTrials.gov identifiers: NCT03333343, NCT03755102).

Histologic transformation to SCLC is another known mechanism of TKI resistance (reported in 15\% of patients after receiving osimertinib). ${ }^{16}$ In these patients, the original EGFR mutation is usually retained, but SCLCs lose their dependence on EGFR activity. ${ }^{10,16}$ Non-EGFR resistance mechanisms to third-generation TKIs occur preferably with T790M loss. ${ }^{16}$ Even after treatment with first- and secondgeneration TKIs, a low ratio of T790M/activating mutation could suggest the presence of other concomitant resistance mechanisms, such as an SCLC transformation. ${ }^{22}$ Indeed, such a ratio could hypothetically indicate the proportion of cells that are T790M positive, assuming that every cancer cell harbors the original EGFR-activating mutation. ${ }^{23}$ Chabon et $\mathrm{a}^{24}$ observed a decrease of the $T 790 \mathrm{M} /$ activating mutation ratio during third-generation TKI therapy and a lower response in patients with a pretreatment ratio of $\leq 0.5$. In the case of SCLC transformation, a favorable response to etoposide and platinum can be expected ${ }^{25}$; therefore, a rebiopsy should be performed when a histologic transformation is suspected (eg, aggressive progression).

In this case report, which describes a representative patient, different subclones harboring distinct resistance mechanisms coexisted. T790M mutation appeared after 9 months of treatment with gefitinib. The initial ratio of T790M/activating mutation was below the threshold of 0.05 and, according to the findings of Chabon et al, ${ }^{24}$ the response to osimertinib was limited to a stable disease by RECIST criteria. This ratio deeply decreased (Table 1) at progression after 12 months of therapy with osimertinib when C797S appeared concomitantly with a small-cell transformation, after a total of 20 months of treatment with TKls. At this time point, we observed a reduction of $T 790 \mathrm{M} /$ del19 ratio in ctDNA, which was similar to the C797S/del19 ratio (Table 1). Because neuroendocrine cancer cells retained EGFR-activating mutation, the increase in allelic frequency of EGFR del19 could be explained in our patient by the SCLC transformation. To our knowledge, this is the first study showing the coexistence of C797S in the rare trans conformation with T790M and SCLC transformation after osimertinib.

No tissue biopsy was performed in available case reports of TKI combination treatment in NSCLC acquiring C797S in the trans conformation; hence, the heterogeneous landscape of cancer evolution might not have been completely assessed, and more data are needed to understand the incidence of the co-occurrence of trans-C797S and SCLC transformation. 
In conclusion, our case report underlines the importance of monitoring the evolution of the disease and of tissue rebiopsy for identifying heterogeneous resistance mechanisms, especially in patients with aggressive progression,

\section{AFFILIATIONS}

${ }^{1}$ Niguarda Cancer Center, Grande Ospedale Metropolitano Niguarda, Milan, Italy

${ }^{2}$ Department of Oncology and Hemato-Oncology, Università degli Studi di Milano, Milan, Italy

\section{CORRESPONDING AUTHOR}

Andrea Sartore-Bianchi, MD, Department of Oncology and HematoOncology, Grande Ospedale Metropolitano Niguarda and Università degli Studi di Milano, Milano, Italy; e-mail: andrea.sartorebianchi@unimi.it.

\section{EQUAL CONTRIBUTION}

E.G.P. and C.L. contributed equally as first authors.

S.M.V., S.S., and A.S.-B. equally contributed as senior authors.

\section{SUPPORT}

E.G.P. was supported by the special grant, Studies to Develop Therapies Against Colorectal Cancer in Young Adults, from Fondazione Oncologia Niguarda Onlus. This study was supported by Fondazione Oncologia Niguarda Onlus.

\section{AUTHOR CONTRIBUTIONS}

Conception and design: Elio G. Pizzutilo, Calogero Lauricella, Giulio Cerea, Massimo Torre, Salvatore Siena, Andrea Sartore-Bianchi Financial support: Salvatore Siena

Provision of study materials or patients: Elio G. Pizzutilo, Calogero Lauricella, Valentina Motta, Spyridon Alexiadis, Angelo Vanzulli, Silvio M. Veronese, Salvatore Siena

Collection and assembly of data: Elio G. Pizzutilo, Calogero Lauricella Giulio Cerea, Laura G. Giannetta, Stefano Stabile, Valentina Motta Spyridon Alexiadis, Angelo Vanzulli, Emanuela Bonoldi, Silvio M. Veronese, Salvatore Siena, Andrea Sartore-Bianchi

Data analysis and interpretation: Elio G. Pizzutilo, Calogero Lauricella, Giulio Cerea, Laura G. Giannetta, Gianluca Tomasello, Valentina Motta, Francesco Scaglione, Emanuela Bonoldi, Silvio M. Veronese, Salvatore Siena, Andrea Sartore-Bianchi

Manuscript writing: All authors

Final approval of manuscript: All authors

Accountable for all aspects of the work: All authors to better define potential treatment modifications. A combination of different therapies might lead to clinical benefit in selected patients harboring different resistant subclones.

\section{AUTHORS' DISCLOSURES OF POTENTIAL CONFLICTS OF INTEREST}

The following represents disclosure information provided by authors of this manuscript. All relationships are considered compensated unless otherwise noted. Relationships are self-held unless noted. I = Immediate Family Member, Inst = My Institution. Relationships may not relate to the subject matter of this manuscript. For more information about ASCO's conflict of interest policy, please refer to www.asco.org/rwc or ascopubs. org/po/author-center.

Open Payments is a public database containing information reported by companies about payments made to US-licensed physicians (Open Payments).

Angelo Vanzulli

Travel, Accommodations, Expenses: Bracco Diagnostics, GE Healthcare

Emanuela Bonoldi

Consulting or Advisory Role: Bayer

Silvio M. Veronese

Honoraria: Bayer, Janssen Cilag

Travel, Accommodations, Expenses: AstraZeneca, Novartis

\section{Salvatore Siena}

Stock and Other Ownership Interests: Ignyta

Honoraria: Amgen, Bayer

Consulting or Advisory Role: Amgen, Genentech, Bayer, Bristol-Myers Squibb, Celgene, Clovis Oncology, Daiichi Sankyo, Incyte, Merck, Novartis, Seattle Genetics

Speakers' Bureau: Amgen, Bayer, Bayer (Inst)

Patents, Royalties, Other Intellectual Property: Amgen

Travel, Accommodations, Expenses: Amgen, Bayer, Roche

Andrea Sartore-Bianchi

Consulting or Advisory Role: Amgen, Bayer, Sanofi

Speakers' Bureau: Amgen, Bayer, Sanofi

Travel, Accommodations, Expenses: Amgen, Bayer, Sanof

No other potential conflicts of interest were reported.

\section{REFERENCES}

1. Hsu W-H, Yang JC-H, Mok TS, et al: Overview of current systemic management of EGFR-mutant NSCLC. Ann Oncol 29: i3-i9, 2018 (suppl 1)

2. Mitsudomi T, Morita S, Yatabe Y, et al: Gefitinib versus cisplatin plus docetaxel in patients with non-small-cell lung cancer harbouring mutations of the epidermal growth factor receptor (WJTOG3405): An open label, randomised phase 3 trial. Lancet Oncol 11:121-128, 2010

3. Rosell R, Carcereny E, Gervais R, et al: Erlotinib versus standard chemotherapy as first-line treatment for European patients with advanced EGFR mutationpositive non-small-cell lung cancer (EURTAC): A multicentre, open-label, randomised phase 3 trial. Lancet Oncol 13:239-246, 2012

4. Sequist LV, Yang JC-H, Yamamoto N, et al: Phase III study of afatinib or cisplatin plus pemetrexed in patients with metastatic lung adenocarcinoma with EGFR mutations. J Clin Oncol 31:3327-3334, 2013

5. Soria J-C, Ohe Y, Vansteenkiste J, et al: Osimertinib in untreated EGFR-mutated advanced non-small-cell lung cancer. N Engl J Med 378:113-125, 2018

6. Westover D, Zugazagoitia J, Cho BC et al: Mechanisms of acquired resistance to first- and second-generation EGFR tyrosine kinase inhibitors. Ann Oncol 29: i10-i19, 2018 (suppl 1)

7. Ricordel C, Friboulet L, Facchinetti F, et al: Molecular mechanisms of acquired resistance to third-generation EGFR-TKIs in EGFR T790M-mutant lung cancer. Ann Oncol 29:i28-i37, 2018 (suppl 1)

8. Yu HA, Tian SK, Drilon AE, et al: Acquired resistance of EGFR-mutant lung cancer to a T790M-specific EGFR inhibitor: Emergence of a third mutation (C797S) in the EGFR tyrosine kinase domain. JAMA Oncol 1:982-984, 2015 
9. Thress KS, Paweletz CP, Felip E, et al: Acquired EGFR C797S mutation mediates resistance to AZD9291 in non-small cell lung cancer harboring EGFR T790M. Nat Med 21:560-562, 2015

10. Niederst MJ, Hu H, Mulvey HE, et al: The allelic context of the C797S mutation acquired upon treatment with third-generation EGFR inhibitors impacts sensitivity to subsequent treatment strategies. Clin Cancer Res 21:3924-3933, 2015

11. Rolfo C, Mack PC, Scagliotti GV, et al: Liquid biopsy for advanced non-small cell lung cancer (NSCLC): A statement paper from the IASLC. J Thorac Oncol 13:1248-1268, 2018

12. Tan DSW, Yom SS, Tsao MS, et al: The International Association for the Study of Lung Cancer consensus statement on optimizing management of EGFR mutation-positive non-small cell lung cancer: Status in 2016. J Thorac Oncol 11:946-963, 2016

13. Jukna A, Montanari G, Mengoli MC, et al: Squamous cell carcinoma "transformation" concurrent with secondary T790M mutation in resistant EGFR-mutated adenocarcinomas. J Thorac Oncol 11:e49-e51, 2016

14. Sequist LV, Waltman BA, Dias-Santagata D, et al: Genotypic and histological evolution of lung cancers acquiring resistance to EGFR inhibitors. Sci TransI Med 3: 75ra26, 2011

15. Yu HA, Arcila ME, Rekhtman N, et al: Analysis of tumor specimens at the time of acquired resistance to EGFR-TKI therapy in 155 patients with EGFR-mutant lung cancers. Clin Cancer Res 19:2240-2247, 2013

16. Oxnard GR, Hu Y, Mileham KF, et al: Assessment of resistance mechanisms and clinical implications in patients with EGFR T790M-positive lung cancer and acquired resistance to osimertinib. JAMA Oncol 4:1527-1534, 2018

17. Yang Z, Yang N, Ou Q, et al: Investigating novel resistance mechanisms to third-generation EGFR tyrosine kinase inhibitor osimertinib in non-small cell lung cancer patients. Clin Cancer Res 24:3097-3107, 2018

18. Leone A: C797S and T790M EGFR mutations in non-small cell lung cancer: In trans or in separate clones? J Thorac Oncol 13:e21-e22, 2018

19. Arulananda S, Do H, Musafer A, et al: Combination osimertinib and gefitinib in C797S and T790M EGFR-mutated non-small cell lung cancer. J Thorac Oncol 12:1728-1732, 2017

20. Wang Z, Yang J-J, Huang J, et al: Lung adenocarcinoma harboring EGFR T790M and in trans C797S responds to combination therapy of first- and thirdgeneration EGFR TKIs and shifts allelic configuration at resistance. J Thorac Oncol 12:1723-1727, 2017

21. Zhou Z, Zhao Y, Shen S et al: Durable clinical response of lung adenocarcinoma harboring EGFR 19Del/T790M/in trans-C797S to combination therapy of firstand third-generation EGFR TKIs. J Thorac Oncol 14:e157-e159, 2019

22. Minari R, Bordi P, Del Re M, et al: Primary resistance to osimertinib due to SCLC transformation: Issue of T790M determination on liquid re-biopsy. Lung Cancer $115: 21-27,2018$

23. Piotrowska Z, Niederst MJ, Karlovich CA, et al: Heterogeneity underlies the emergence of EGFRT790 wild-type clones following treatment of T790M-positive cancers with a third-generation EGFR inhibitor. Cancer Discov 5:713-722, 2015

24. Chabon JJ, Simmons AD, Lovejoy AF, et al: Circulating tumour DNA profiling reveals heterogeneity of EGFR inhibitor resistance mechanisms in lung cancer patients. Nat Commun 7:11815, 2016 [Erratum: Nat Commun 7:13513, 2016]

25. Marcoux N, Gettinger SN, O'Kane G, et al: EGFR-mutant adenocarcinomas that transform to small-cell lung cancer and other neuroendocrine carcinomas: Clinical outcomes. J Clin Oncol 37:278-285, 2019 